\title{
PA during the COVID-19 outbreak in China: a cross-sectional study
}

\author{
Yingjun $\mathrm{Nie}^{1} \cdot$ Yuanyan $\mathrm{Ma}^{2} \cdot$ Xiaodong $\mathrm{Li}^{3} \cdot$ Yankong $\mathrm{Wu}^{4} \cdot$ Weixin $\mathrm{Liu}^{4} \cdot$ Zhenke $\operatorname{Tan}^{4} \cdot$ \\ Jiahui $\mathrm{Li}^{4} \cdot$ Ce Zhang ${ }^{4} \cdot$ Chennan $\mathrm{Lv}^{4} \cdot$ Ting Liu $^{4}$
}

Received: 20 May 2021 / Accepted: 9 September 2021

(c) The Author(s), under exclusive licence to Springer-Verlag London Ltd., part of Springer Nature 2021

\begin{abstract}
COVID-19 has undergone several mutations and is still spreading in most countries now. PA has positive benefits in the prevention of COVID-19 infection and counteracting the negative physical and mental effects caused by COVID-19. However, relevant evidence has indicated a high prevalence of physical inactivity among the general population, which has worsened due to the outbreak of the pandemic, and there is a severe lack of exercise guidance and mitigation strategies to advance the knowledge and role of PA to improve physical and mental health in most countries during the epidemic. This study surveyed the effects of COVID-19 on PA in Chinese residents during the pandemic and provided important reference and evidence to inform policymakers and formulate policies and planning for health promotion and strengthening residents' PA during periods of public health emergencies. ANOVA, Kolmogorov-Smirnov, the chi-square test and Spearman correlation analysis were used for statistical analysis. A total of 14,715 participants were included. The results show that nearly $70 \%$ of Chinese residents had inadequate PA (95\%CI 58.0\%-82.19\%) during the COVID-19 outbreak, which was more than double the global level $(27.5 \%, 95 \% \mathrm{CI} 25.0 \%-32.2 \%)$. The content, intensity, duration, and frequency of PA were all affected during the period of home isolation, and the types of PA may vary among different ages. The lack of physical facilities and cultural environment is the main factor affecting PA. However, there was no significant correlation between insufficient PA and the infection rate. During the period of home isolation and social distance of epidemic prevention, it is necessary to strengthen the scientific remote network monitoring and guidance for the process of PA in China.
\end{abstract}

Keywords COVID-19 - Chinese residents · PA · Influence $\cdot$ Home isolation · Social distancing $\cdot$ Health well-being PA guidance

\section{Introduction}

COVID-19 has become a major global public health emergency and led to over 180 million confirmed cases and over 4 million deaths globally as of 12th Jul 2021,

Yingjun Nie and Yuanyan Ma contributed equally to this work and share first authorship.

Xiaodong Li

lxd@hhtc.edu.cn

1 Art of School, Wuhan Sports University, Wuhan 430079, China

2 College of Science and Technology, Wuhan Sports University, Wuhan 430079, China

3 Smart Health Promote Laboratory, Huaihua University, Huaihua 418000, China

4 Graduate School, Wuhan Sports University, Wuhan 430079, China including 92,066 confirmed cases and 4636 deaths in China [1]. The World Health Organization (WHO) has declared the global need for a long-term outbreak response. At present, there are still many doubts and uncertainties in the transmission, infection and treatment of COVID-19, and the best way to prevent and cure COVID-19 is to prevent infection and improve self-immunity [2]. An overwhelming body of evidence has demonstrated the positive benefits of engaging in adequate PA on enhancing the immunity [3-5] to reduce the risk of infectious diseases (such as viral and bacterial infections) and have positive effects on preventing COVID-19 infection and counteracting the negative physical and mental effects caused by COVID-19 
Table 1 Distribution of respondents in provinces and cities

\begin{tabular}{llllll}
\hline Provinces & Count & Provinces & Count & Provinces & Count \\
\hline Bei jing & 408 & An hui & 518 & Gui zhou & 385 \\
Tian jin & 450 & Fu jian & 657 & Yun nan & 455 \\
He bei & 393 & Jiang xi & 687 & Tibet & 301 \\
Shan xi & 433 & He nan & 739 & Chong qing & 285 \\
Inner Mongolia & 336 & Hu bei & 824 & Shan xi & 359 \\
Liao ning & 455 & Hu nan & 780 & Gan su & 351 \\
Ji lin & 453 & Guang dong & 742 & Qing hai & 389 \\
Hei long jiang & 302 & Guang xi & 483 & Ning xia & 437 \\
Jiang su & 583 & Hai nan & 489 & Xin jiang & 240 \\
Zhe jiang & 406 & Si chuan & 507 & & \\
Shang hai & 386 & Shan dong & 482 & & \\
\hline
\end{tabular}

Table 2 Age of the respondents

\begin{tabular}{lll}
\hline Age (years) & Frequency & Percent \\
\hline$\leq 17$ & 1258 & $8.5 \%$ \\
$18-24$ & 4180 & $28 \%$ \\
$25-29$ & 2154 & $15 \%$ \\
$30-34$ & 1615 & $11 \%$ \\
$35-39$ & 1756 & $12 \%$ \\
$40-44$ & 1704 & $12 \%$ \\
$45-49$ & 920 & $6 \%$ \\
$50-54$ & 440 & $3 \%$ \\
$55-59$ & 228 & $2 \%$ \\
$60-69$ & 460 & $3 \%$ \\
\hline
\end{tabular}

Table 3 Education level of the respondents

\begin{tabular}{lcc}
\hline Education level & Frequency & Percent \\
\hline $\begin{array}{l}\text { Primary school and below (including no } \\
\text { systematic education) }\end{array}$ & 753 & $5 \%$ \\
$\quad \begin{array}{l}\text { Junior high school } \\
\text { High school (higher vocational school, }\end{array}$ & 1630 & $11 \%$ \\
$\quad$ technical secondary school, technical school) & 1873 & $13 \%$ \\
$\begin{array}{l}\text { Junior college (self-examination, adult } \\
\quad \text { education, promotion) }\end{array}$ & 3176 & $22 \%$ \\
$\begin{array}{l}\text { Bachelor's degree } \\
\text { Master degree or above }\end{array}$ & 4909 & $33 \%$ \\
\hline
\end{tabular}

[6-8]. WHO also is continuously advising to strengthen PA as the effective method to prevent infection of COVID-19.

Relevant evidence has indicated a high prevalence of physical inactivity among the general population which has worsened due to the outbreak of the pandemic [9], such as a global survey of six continents has shown that all intensity levels of PA during new coronary pneumonia are negatively affected [10], and social distancing and home quarantine measures have caused dramatic decreased PA and increased sedentary time [11]. However, insufficient PA is a key risk factor for cardiovascular diseases, hypertension, and diabetes $[12,13]$, and sedentary muscle fixation and other muscle fixation will destroy and worsen mitochondrial homeostasis, leading to organic and systemic inflammation, which is also an important mechanism of COVID-19 [14]. Recent studies also have shown that there is a severe lack of PA guidance and mitigation strategies to advance the knowledge and role of PA to improve physical and mental health in most countries during the epidemic [15]. On the whole, existing studies have mainly revealed this phenomenon that there was a high prevalence of physical inactivity due to the outbreak of the pandemic and analyzed the importance of strengthening proper PA to promote physical and mental health and prevent epidemics [16-18]. However, a series of crucial questions need to be answered: Is the PA of all groups affected during the epidemic? What forms of PA are selected by different groups during the epidemic? What factors have caused the increase of insufficient PA during the epidemic? Then, the lack of large samples made it difficult to explain these crucial questions.

From January 24 to April 22, 2020, the Chinese government adopted a series of prevention and control strategies, such as city closure, home isolation, social distance, and prohibition of tourism so that the epidemic situation could be effectively controlled in three months [19]. This situation in China may offer a relatively stable research environment and a huge amount of samples for this study due to the essential research question is the relationship between PA and COVID-19. Therefore, the aim of this study was to investigate the states and characteristics of PA among Chinese residents to help people reap the great 
Table 4 Professional of the respondents

\begin{tabular}{lrr}
\hline Professional & Frequency & Percent \\
\hline Front-line medical staff for epidemic prevention & 520 & $4 \%$ \\
Front-line epidemic prevention volunteers & 599 & $4 \%$ \\
Public institution/civil servant/government staff & 1559 & $11 \%$ \\
Professionals (e.g., teachers/lawyers, etc.) & 1538 & $11 \%$ \\
Service personnel (e.g., catering waiter/driver/salesperson, etc.) & 742 & $5 \%$ \\
Freelancer (writer/artist/tour guide, etc.) & 694 & $5 \%$ \\
Workers (factory workers/construction workers/city sanitation workers, etc.) & 674 & $5 \%$ \\
Company employees & 1516 & $11 \%$ \\
Businessman/employer/peddler/self-employed & 620 & $4 \%$ \\
Students & 3005 & $21 \%$ \\
A housewife & 801 & $6 \%$ \\
Farmer/herdsman/fisherman & 1154 & $8 \%$ \\
Unemployed/unemployed & 997 & $7 \%$ \\
\hline
\end{tabular}

Table 5 Insufficient rate of PA during the outbreak period in China

\begin{tabular}{|c|c|c|c|c|c|}
\hline \multicolumn{2}{|c|}{ Total of participants $(n=14,715)$} & \multicolumn{2}{|l|}{$\operatorname{Male}(n=6894)$} & \multicolumn{2}{|l|}{$\operatorname{Female}(n=7821)$} \\
\hline $\begin{array}{l}\text { Total of } \\
\text { participants with } \\
\text { insufficient PA }\end{array}$ & Rate $(95 \% \mathrm{CI})$ & $\begin{array}{l}\text { Total of male } \\
\text { participants with } \\
\text { insufficient PA }\end{array}$ & Rate $(95 \% \mathrm{CI})$ & $\begin{array}{l}\text { Total of female } \\
\text { participants with } \\
\text { insufficient PA }\end{array}$ & Rate $(95 \% \mathrm{CI})$ \\
\hline 10,317 & $70.11 \%(58.03-82.19)$ & 4308 & $62.49 \%(45.27-79.71)$ & 6009 & $76.83 \%(61.96-91.71)$ \\
\hline
\end{tabular}

benefits of regular PA on health well-being and preventing COVID-19 infection during the pandemic.

The major contributions of the proposed work are observed as:

- Surveying the effects of COVID-19 on PA may provide important reference and evidence to inform policymakers and formulate policies and planning for health promotion and strengthening residents' PA during the periods of public health emergencies.

- Revealing the main affecting factors of the insufficient PA rate helps to understand how to create conditions to

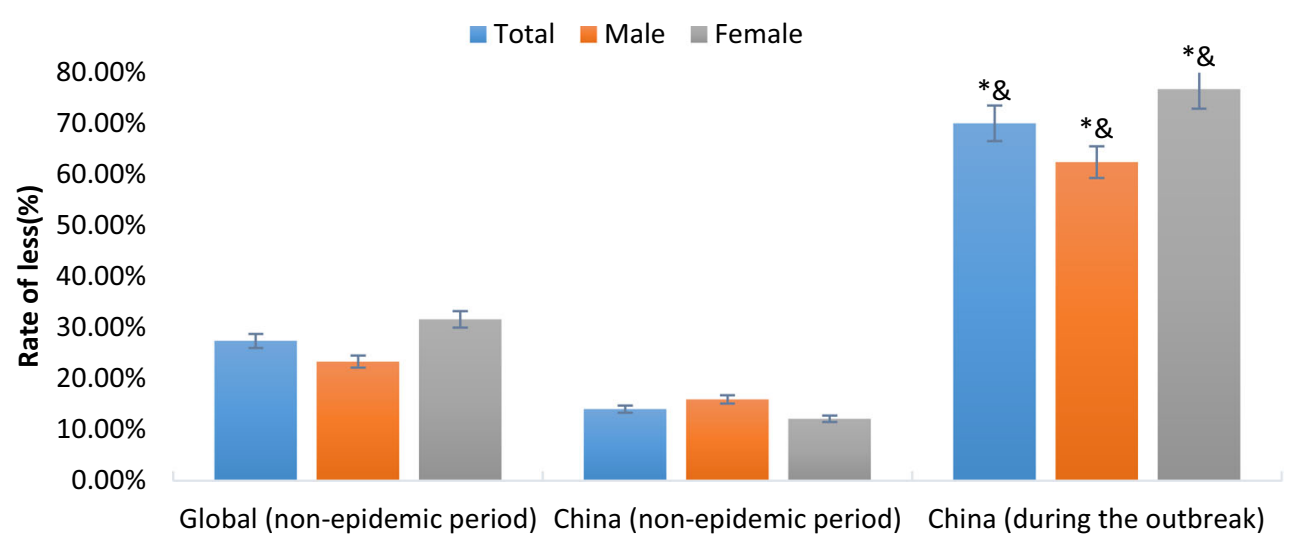

Fig. 1 The rates of physical inactivity among global and Chinese residents in non-epidemic period and in the outbreak period. $*$ Means $p<0.05$ versus global level; and means $p<0.05$ versus China without COVID-19 outbreak (non-epidemic period) 
Fig. 2 Sperm diagram of the relationship between the rate of physical inactivity of residents and the rate of COVID-19 infection in all provinces and cities

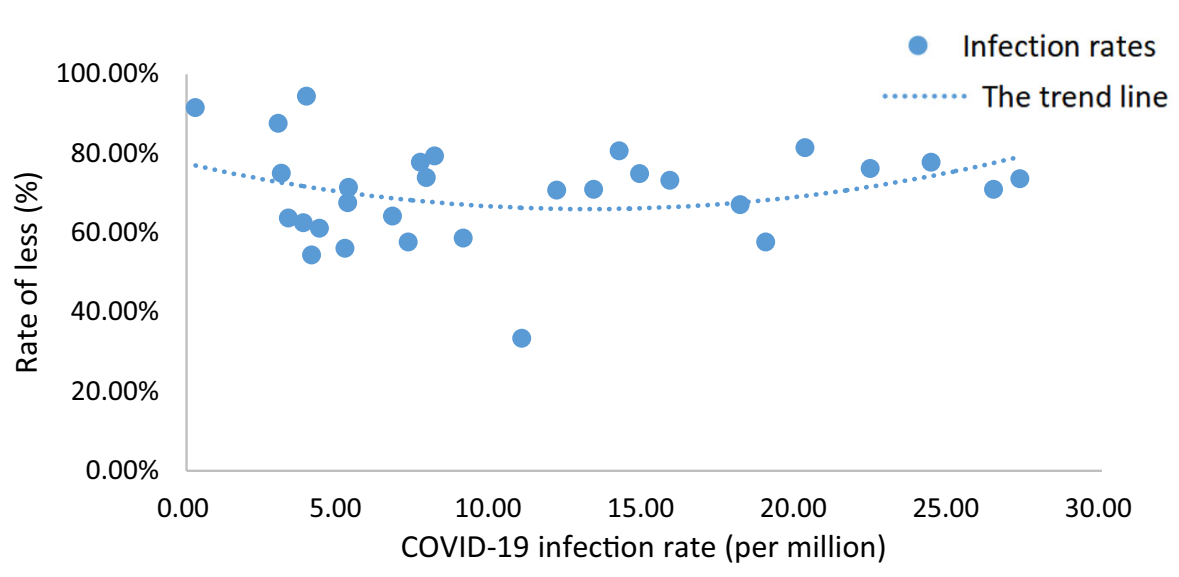

improve PA during the period of home isolation and social distance of epidemic prevention.

- Understanding the differences in PA content, intensity, duration, and frequency at all age groups is beneficial for adopting corresponding PA intervention methods and forms to promote different groups' physical and mental health during the periods of public health emergencies.

The remaining paper is structured as follows: Sect. 2 provides detail of survey materials and methods. Section 3 first presents the insufficient PA rate and the main factors influencing PA and then gives characteristics and schemes of PA in different groups. Section 4 gives a detailed discussion about survey results and the limitations and prospects of research. Finally, the conclusions of this study are presented in Sect. 5.

\section{Methods}

\subsection{The study population}

According to the definition of the development stage of the epidemic in China by the Chinese Health Commission, the pandemic period in China is from January 24 to April 22, 2020. The study recruited Chinese residents living in 31 provinces of China (Table 1) covered by the physical fitness monitoring points system during the period. Since China has adopted unified isolation management measures for people infected with COVID-19, the subjects of the investigation were those who were not infected with COVID-19. The participants were divided into the following ten groups based on their age: 17 years old and below, 18-24 years old, 25-29 years old, 30-34 years old, 35-39 years old, 40-44 years old, 45-49 years old, 50-54 years old, 55-59 years old, 60 years old, and above (Table 2). The

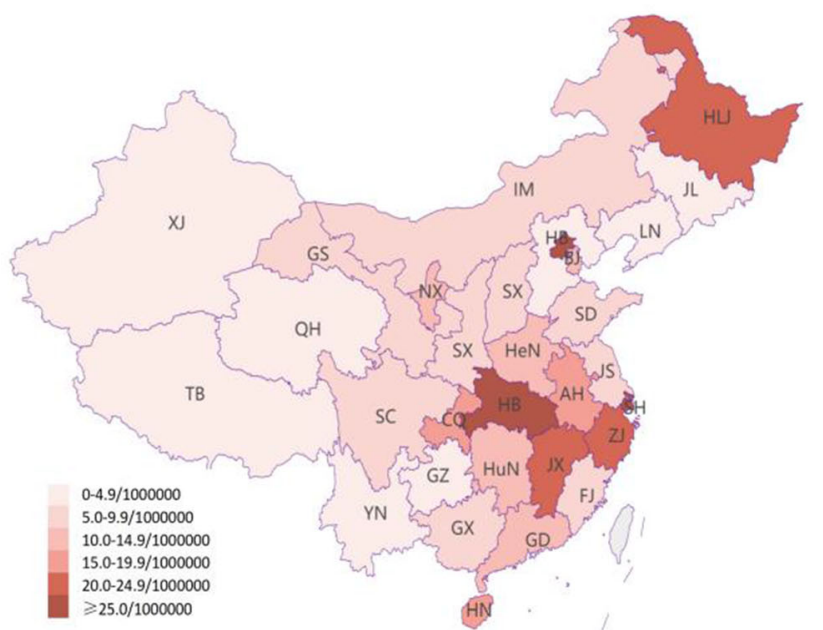

COVID-19 infection rate

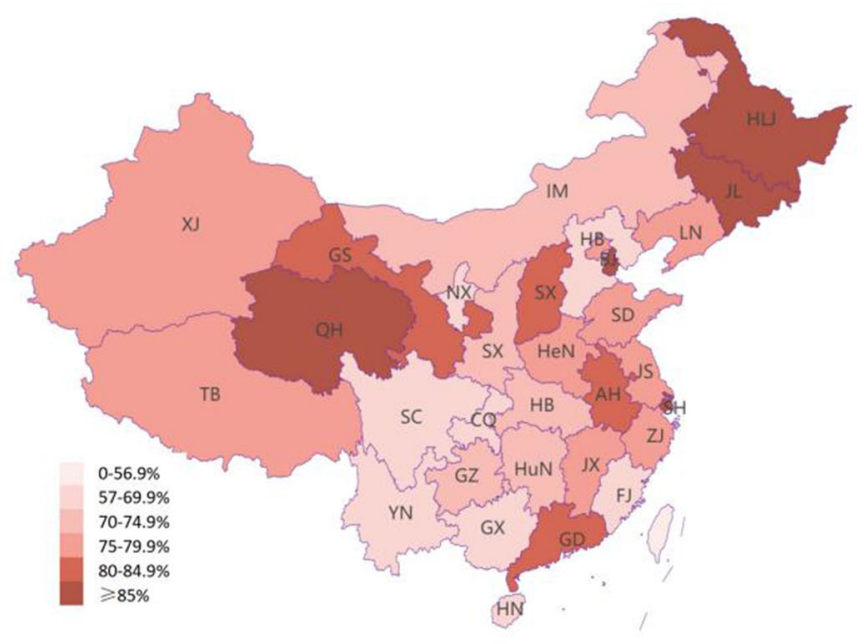

Inadequate $\mathrm{PA}$ rate

Fig. 3 Covid-19 infection rate and inadequate PA rate of residents in each province and city 
Fig. 4 Scatter diagram of the relationship between insufficient PA rate and COVID-19 infection rate of urban residents with more severe infection

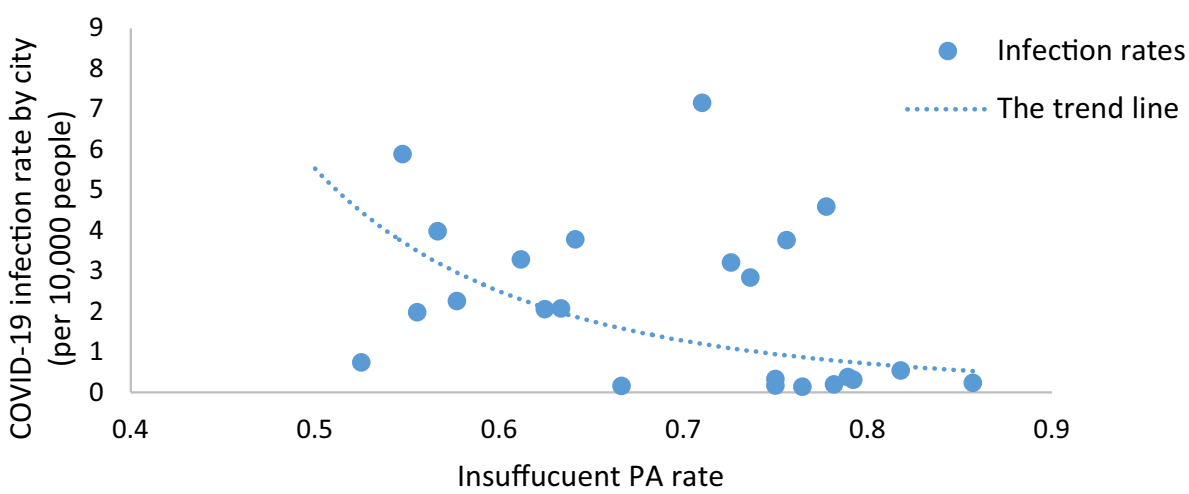

survey also included social factors such as gender, age, education level (Table 3), geographic location (province and city), occupation (Table 4), and region (town and village) of the participants.

\subsection{Survey methodology}

The questionnaire was distributed using a snowball sampling strategy to recruit questionnaire respondents due to the recruitment pool of participants and was distributed via the WeChat and social media platforms with high clickthrough rates and usage rates of all groups. In addition, instructors in social sports in various communities, cities, and provinces participated in questionnaire distribution via their working platforms. Through these ways, ensure that residents from different regions of the country participated and maximize the diversity and representativeness of the population participating in the survey. Individuals were asked to record their PA during the
COVID-19 pandemic, within an overall collection period from 22 June to 20 July 2020. The information (PA status) included the content, intensity, weekly frequency, duration, exercise monitoring style, and influencing factors of various PA.

The PA data were obtained by using the International PA Questionnaire (IPAQ), which was verified in China, and then managed and filtered logically [20]. The Cronbach alpha coefficient of the questionnaire in this study was 0.89 , the retest reliability was also above 0.7 , and the internal consistency was 0.87 . The form asks participants to record the content, intensity, weekly frequency, duration, and exercise monitoring style of various physical activities that they engage in weekly and daily, as well as the factors that affect PA during the pandemic. According to the IPAQ guidelines for data processing and analysis [21], and the International Health Organization standard of $150 \mathrm{~min}$ per week of moderate intensity or $75 \mathrm{~min}$ per week of high intensity, or any equivalent combination of

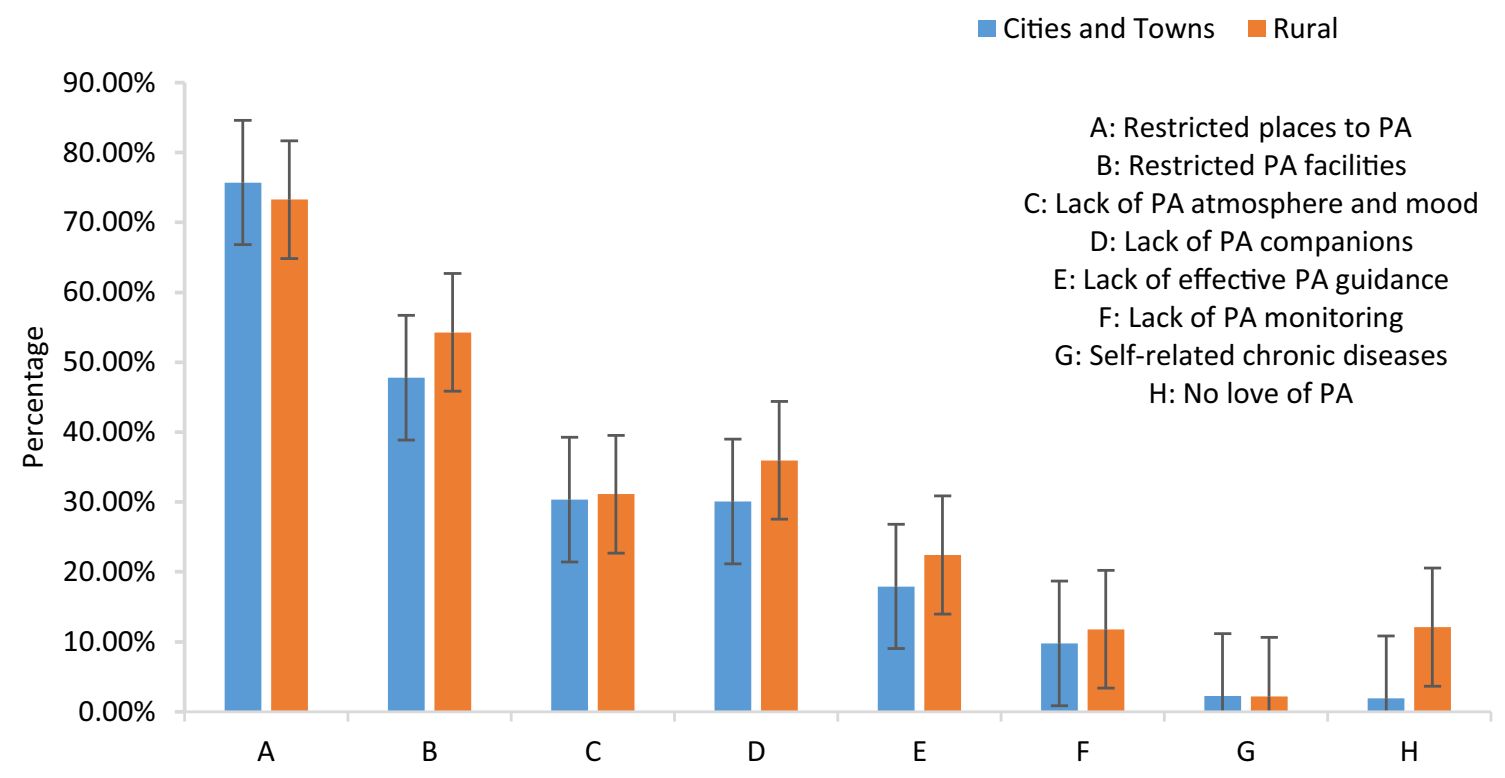

Fig. 5 Reasons influencing PA in urban and rural areas 
Table 6 ANOVA of weekly PA time of people with different education levels

\begin{tabular}{lll}
\hline Education level & Exercise time per week $(\mathrm{M} \pm \mathrm{SD})$ & Insufficient PA rate \\
\hline Primary school and below (including no systematic education) $(n=753)$ & $65.94 \pm 39.71$ & $90.65 \%$ \\
Junior high school $(n=1630)$ & $117.07 \pm 119.15$ & $77.04 \%$ \\
High school $(n=1873)$ & $84.47 \pm 105.43$ & $80.09 \%$ \\
Junior college $(n=3176)$ & $104.47 \pm 142.94$ & $74.24 \%$ \\
Bachelor's degree $(n=4909)$ & $136.97 \pm 165.55$ & $57.93 \%$ \\
Master degree or above $(n=2374)$ & $127.96 \pm 138.99$ & $56.28 \%$ \\
F & 47.666 & \\
$\mathrm{p}$ & $0.000^{* *}$ & \\
\hline
\end{tabular}

$M=$ mean; $S D=$ standard deviation

*Means $p<0.05$; **means $p<0.01$

the two [22], less than this is considered to be insufficient PA.

\subsection{Statistical analysis}

All data were analyzed with SPSS software 26.0 (IBM Inst., Chicago, IL, USA). The weekly total duration of PA was calculated. Descriptive statistics such as percentages, 95\% CIs, means, and standard deviations were calculated for categorical variables and continuous variables to reflect the demographic characteristics of the survey population. The $\mathrm{X}^{2}$ test was used to assess statistical differences in rates of physical inactivity by residential area (urban and rural areas), sex (men and women), and age group. Kolmogorov-Smirnov was used to test the normality of continuous variables. Spearman correlation coefficient was used to evaluate the relationship between COVID-19 infection rate and average physical inactivity rate in each province and city. ANOVA was used to analyze the differences in PA content, content source, exercise form, and monitoring among people with different education levels.

A Chi-square test was used to test the differences among different age groups on the variables of PA intensity, duration and frequency. Hypothesis: (1) there is no significant difference in PA insufficiency rate between urban and rural; (2) there is no significant difference between in PA insufficiency rate between men and women; (3) there is a significant difference in PA in different age groups. According to the number of independent variables, the degree of freedom was determined to be the sample size minus 3. And the significant level $\alpha=0.05$ was selected. Finally, the statistic and critical values were compared and determined whether to accept these hypotheses or not.
Fig. 6 Distribution of PA insufficiency rate in different age groups

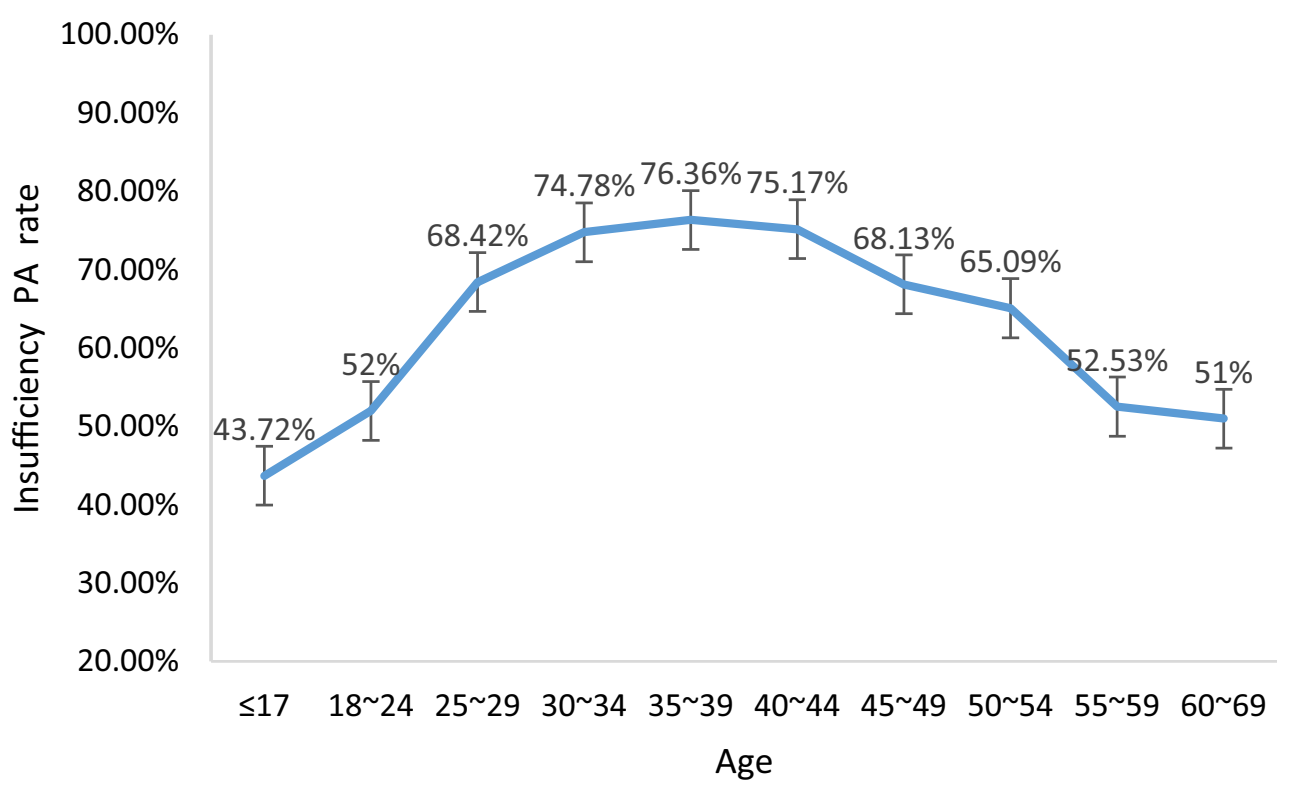




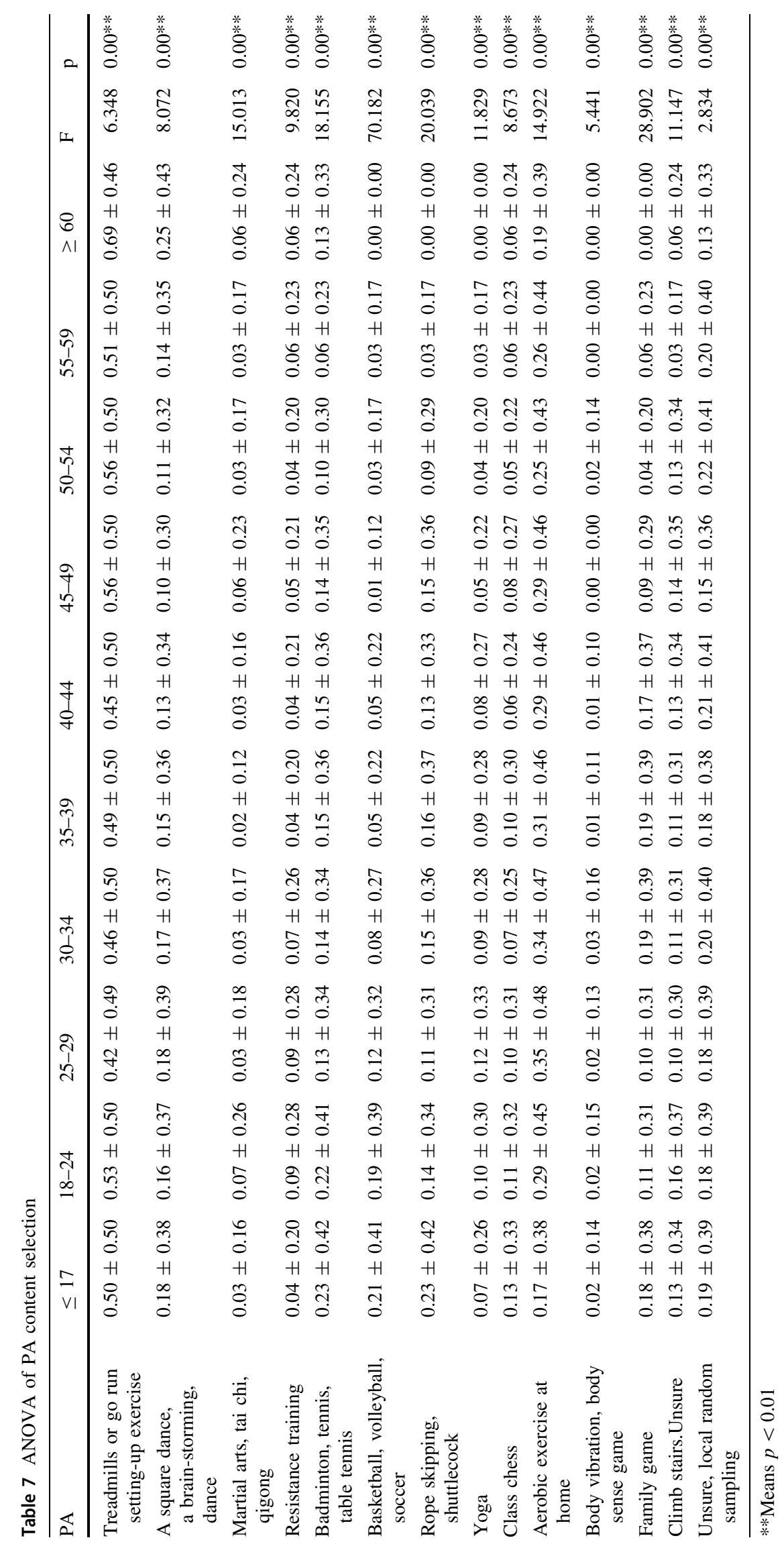


Table 8 ANOVA of PA content sources of people of different age

\begin{tabular}{llllllll}
\hline Age & $\begin{array}{l}\text { Usual exercise } \\
\text { content }\end{array}$ & $\begin{array}{l}\text { Study and imitate family } \\
\text { members }\end{array}$ & $\begin{array}{l}\text { Local } \\
\text { materials }\end{array}$ & $\begin{array}{l}\text { Study network } \\
\text { video }\end{array}$ & $\begin{array}{l}\text { Consult } \\
\text { books }\end{array}$ & $\begin{array}{l}\text { Friends } \\
\text { recommend }\end{array}$ & $\begin{array}{l}\text { Social organizations } \\
\text { recommend }\end{array}$ \\
\hline$\leq 17$ & $0.55 \pm 0.50$ & $0.22 \pm 0.41$ & $0.36 \pm 0.48$ & $0.33 \pm 0.47$ & $0.12 \pm 0.32$ & $0.11 \pm 0.31$ & $0.10 \pm 0.30$ \\
$18 \sim 24$ & $0.62 \pm 0.49$ & $0.15 \pm 0.36$ & $0.31 \pm 0.46$ & $0.41 \pm 0.49$ & $0.08 \pm 0.27$ & $0.12 \pm 0.33$ & $0.05 \pm 0.22$ \\
$25 \sim 29$ & $0.58 \pm 0.49$ & $0.07 \pm 0.25$ & $0.34 \pm 0.47$ & $0.39 \pm 0.49$ & $0.07 \pm 0.25$ & $0.08 \pm 0.28$ & $0.03 \pm 0.18$ \\
$30 \sim 34$ & $0.54 \pm 0.50$ & $0.12 \pm 0.32$ & $0.30 \pm 0.46$ & $0.45 \pm 0.50$ & $0.09 \pm 0.29$ & $0.11 \pm 0.31$ & $0.03 \pm 0.18$ \\
$35 \sim 39$ & $0.48 \pm 0.50$ & $0.11 \pm 0.31$ & $0.40 \pm 0.49$ & $0.39 \pm 0.49$ & $0.05 \pm 0.22$ & $0.09 \pm 0.28$ & $0.04 \pm 0.19$ \\
$40 \sim 44$ & $0.49 \pm 0.50$ & $0.18 \pm 0.39$ & $0.32 \pm 0.47$ & $0.37 \pm 0.48$ & $0.04 \pm 0.21$ & $0.12 \pm 0.33$ & $0.03 \pm 0.17$ \\
$45 \sim 49$ & $0.58 \pm 0.49$ & $0.12 \pm 0.33$ & $0.33 \pm 0.47$ & $0.31 \pm 0.46$ & $0.07 \pm 0.26$ & $0.10 \pm 0.29$ & $0.05 \pm 0.22$ \\
$50 \sim 54$ & $0.66 \pm 0.47$ & $0.18 \pm 0.38$ & $0.33 \pm 0.47$ & $0.16 \pm 0.37$ & $0.06 \pm 0.24$ & $0.08 \pm 0.28$ & $0.03 \pm 0.17$ \\
$55 \sim 59$ & $0.83 \pm 0.38$ & $0.06 \pm 0.23$ & $0.31 \pm 0.47$ & $0.11 \pm 0.32$ & $0.06 \pm 0.23$ & $0.06 \pm 0.23$ & $0.03 \pm 0.17$ \\
$\geq 60$ & $0.81 \pm 0.39$ & $0.06 \pm 0.24$ & $0.19 \pm 0.39$ & $0.06 \pm 0.24$ & $0.00 \pm 0.00$ & $0.13 \pm 0.33$ & $0.06 \pm 0.24$ \\
$\mathrm{~F}$ & 33.190 & 24.209 & 9.749 & 41.815 & 11.202 & 4.535 & 10.617 \\
$\mathrm{p}$ & $0.000^{* *}$ & $0.000^{* *}$ & $0.000^{* *}$ & $0.000^{* *}$ & $0.000^{* *}$ & $0.000^{* *}$ & $0.000^{* *}$
\end{tabular}

**Means $p<0.01$

Table 9 PA intensity of Chinese residents during COVID-19 outbreak
Table 10 Statistical table of single duration of PA for Chinese residents during the COVID-19 outbreak

\begin{tabular}{llllllll}
\hline Age & High strength & \multicolumn{3}{l}{ Moderate intensity } & Low intensity & Sig \\
\hline$\leq 17$ & 194 & $(19.78 \%)$ & 418 & $(42.61 \%)$ & 369 & $(37.61 \%)$ & $<0.001$ \\
$18 \sim 24$ & 783 & $(21.39 \%)$ & 1603 & $(41.59 \%)$ & 1403 & $(37.03 \%)$ & \\
$25 \sim 29$ & 168 & $(9.15 \%)$ & 956 & $(52.71 \%)$ & 702 & $(40.14 \%)$ & \\
$30 \sim 34$ & 105 & $(7.61 \%)$ & 640 & $(46.38 \%)$ & 635 & $(46.01 \%)$ & \\
$35 \sim 39$ & 68 & $(4.49 \%)$ & 780 & $(51.45 \%)$ & 660 & $(44.06 \%)$ & \\
$40 \sim 44$ & 76 & $(4.92 \%)$ & 912 & $(59.07 \%)$ & 566 & $(36.01 \%)$ & \\
$45 \sim 49$ & 44 & $(5.29 \%)$ & 304 & $(58.17 \%)$ & 484 & $(36.54 \%)$ & \\
$50 \sim 54$ & 24 & $(6.19 \%)$ & 240 & $(53.96 \%)$ & 124 & $(38.86 \%)$ & \\
$55 \sim 59$ & 6 & $(2.86 \%)$ & 84 & $(40.00 \%)$ & 120 & $(57.14 \%)$ & \\
$\geq 60$ & 0 & $(0.00 \%)$ & 184 & $(38.00 \%)$ & 184 & $(62.00 \%)$ & \\
Total & $1468(11.44 \%)$ & $6121(47.69 \%)$ & $5247(40.88 \%)$ & & & & \\
\hline
\end{tabular}

Sig. value was calculated by the chi-square test and represents the difference between the variables

\begin{tabular}{rrrrrr}
\hline Age & \multicolumn{1}{c}{$<30 \min$} & \multicolumn{1}{c}{$30-45 \min$} & \multicolumn{1}{c}{$45-60 \min$} & $60-90 \min$ & $>90 \min$ \\
\hline$\leq 17$ & $271(27.62 \%)$ & $273(27.83 \%)$ & $167(17.02 \%)$ & $178(18.14 \%)$ & $92(9.38 \%)$ \\
$18-24$ & $1017(26.84 \%)$ & $1155(30.48 \%)$ & $869(22.93 \%)$ & $564(14.89 \%)$ & $184(4.86 \%)$ \\
$25-29$ & $726(39.54 \%)$ & $594(32.35 \%)$ & $330(17.97 \%)$ & $174(9.48 \%)$ & $12(0.65 \%)$ \\
$30-34$ & $590(42.75 \%)$ & $440(31.88 \%)$ & $240(17.39 \%)$ & $75(5.43 \%)$ & $35(2.54 \%)$ \\
$35-39$ & $692(45.65 \%)$ & $488(32.19 \%)$ & $248(16.36 \%)$ & $76(5.01 \%)$ & $12(0.79 \%)$ \\
$40-44$ & $748(48.45 \%)$ & $448(29.02 \%)$ & $252(16.32 \%)$ & $80(5.18 \%)$ & $16(1.04 \%)$ \\
$45-49$ & $340(40.87 \%)$ & $232(27.88 \%)$ & $176(21.15 \%)$ & $56(5.18 \%)$ & $28(1.04 \%)$ \\
$50-54$ & $148(38.14 \%)$ & $96(24.74 \%)$ & $84(21.65 \%)$ & $48(12.37 \%)$ & $12(3.09 \%)$ \\
$55-59$ & $66(31.43 \%)$ & $78(37.14 \%)$ & $5(2.38 \%)$ & $12(5.71 \%)$ & $11(5.2 \%)$ \\
$\geq 60$ & $138(37.50 \%)$ & $69(18.75 \%)$ & $115(31.25 \%)$ & $46(12.50 \%)$ & $30(8.15 \%)$ \\
Total & $4736(36.9 \%)$ & $3873(30.17 \%)$ & $2486(19.37 \%)$ & $1309(10.2 \%)$ & $432(3.37 \%)$ \\
\hline
\end{tabular}


Table 11 PA frequency of Chinese residents during COVID-19 outbreak

\begin{tabular}{rllll}
\hline Age & 1-2 Times a week & 3-5 Times a week & 5 Times above a week & Total \\
\hline$\leq 17$ & $409(6.35 \%)$ & $361(7.76 \%)$ & $211(12.09 \%)$ & $981(7.64 \%)$ \\
$18-24$ & $1972(30.60 \%)$ & $1369(29.42 \%)$ & $448(25.67 \%)$ & $3789(29.50 \%)$ \\
$25-29$ & $1020(15.83 \%)$ & $636(13.67 \%)$ & $180(10.32 \%)$ & $1836(14.29 \%)$ \\
$30-34$ & $830(12.88 \%)$ & $370(7.95 \%)$ & $180(10.32 \%)$ & $1380(10.74 \%)$ \\
$35-39$ & $780(12.10 \%)$ & $548(11.77 \%)$ & $188(10.77 \%)$ & $1516(11.80 \%)$ \\
$40-44$ & $752(11.67 \%)$ & $604(12.98 \%)$ & $188(10.77 \%)$ & $1544(12.02 \%)$ \\
$45-49$ & $336(5.21 \%)$ & $372(7.99 \%)$ & $124(7.11 \%)$ & $832(6.48 \%)$ \\
$50-54$ & $188(2.92 \%)$ & $144(3.09 \%)$ & $56(3.21 \%)$ & $388(3.02 \%)$ \\
$55-59$ & $66(1.02 \%)$ & $66(1.42 \%)$ & $78(4.47 \%)$ & $210(1.64 \%)$ \\
$\geq 60$ & $92(1.43 \%)$ & $184(3.95 \%)$ & $92(5.27 \%)$ & $368(2.87 \%)$ \\
Total & 6445 & 4654 & 1745 & 12,844 \\
\hline
\end{tabular}

The COVID-19 infection rate was calculated by dividing the total number of confirmed COVID-19 cases in each province (as of April 22, 2020) as reported by the National Health Commission by the total population of each province (the provincial population at the end of 2019 as quoted in the Statistical Yearbook of China published by the National Bureau of Statistics of China). The parameters of physical inactivity rate during non-epidemic periods were based on the global age-standardized physical inactivity rate published by WHO in 2018 [23], in which the global age-standardized rate of insufficient PA was $27.5 \%$ (95\% CI $25.0 \%-32.2 \%$ ), for males $23.4 \%$ (95\%CI $21.1 \%$ $30.7 \%$ ) and $31.7 \%$ for females (95\%CI $28.6 \%-39.0 \%$ ); The rate of insufficient PA among Chinese residents during the non-epidemic period was $14.1 \%$ (95\%CI $10.1 \%$ $19.4 \%$ ) overall, $16.0 \%$ (95\%CI $11.7 \%-21.4 \%$ ) for men, and $12.2 \%$ (95\% CI $8.5 \%-17.3 \%$ ) for females.

\section{Results}

\subsection{Survey respondents}

During the COVID-19 pandemic, a total of 14,715 participants were included in the final analysis, covering 31 provincial administrative regions in mainland China. A total of 14,715 participants were included $53 \%$ males and $47 \%$ females, and 55\% from cities, and $45 \%$ from rural areas.

During the outbreak period in Covid-19, the insufficient rate of PA among Chinese residents was $70.11 \%$ (95\% CI $58.03 \%-82.19 \%$ ) as shown in Table 5 and Fig. 1, which was more than doubled compared with the global insufficient rate of PA reported by WHO $\left(x^{2}=13,088.10\right.$, $p<0.0001)$. Compared with the rate of insufficient PA
(14.1\%) of Chinese residents during the non-epidemic period, it increased about 5 times $\left(x^{2}=12,700.00\right.$, $p<0.0001)$.

\subsection{Correlation analysis between PA and COVID- 19 infection rate of residents in different regions}

The test results of the normal distribution of the two groups of variables of PA insufficiency rate and COVID-19 infection rate in each province showed a significance greater than 0.05 , and the data followed the normal distribution. Therefore, Pearson correlation analysis was used to determine whether the PA insufficiency rate and COVID-19 infection rate in each province met the linear relationship. The results showed that the Pearson correlation coefficient was $-0.023, R^{2}=0.0833$, and the SIG value was $0.896>0.05$, indicating that the correlation between the two groups of variables was not significant. So there was no significant correlation between the COVID-19 infection rate in each province and the PA insufficiency rate in the province, as shown in Figs. 2 and 3.

Further, in view of the serious difference in the degree of infection among provinces and cities, the correlation between the degree of infection and the rate of physical inactivity in cities with more severe epidemic period was further analyzed by taking cities with more severe epidemic period as an example. According to the data from the National Health Commission, cities in the key epidemic areas during the COVID-19 outbreak period include cities in Hubei Province (Wuhan, Xiaogan, Huanggang, Jingzhou, Ezhou, Suizhou, Xiangyang, Huangshi, Yichang, Jingmen, Xianning, Shiyan, Xiantao, Tianmen, Enshi, Qianjiang); Wenzhou, Taizhou, Hangzhou, and Ningbo, Zhejiang Province; Xinyang City, Henan Province, 


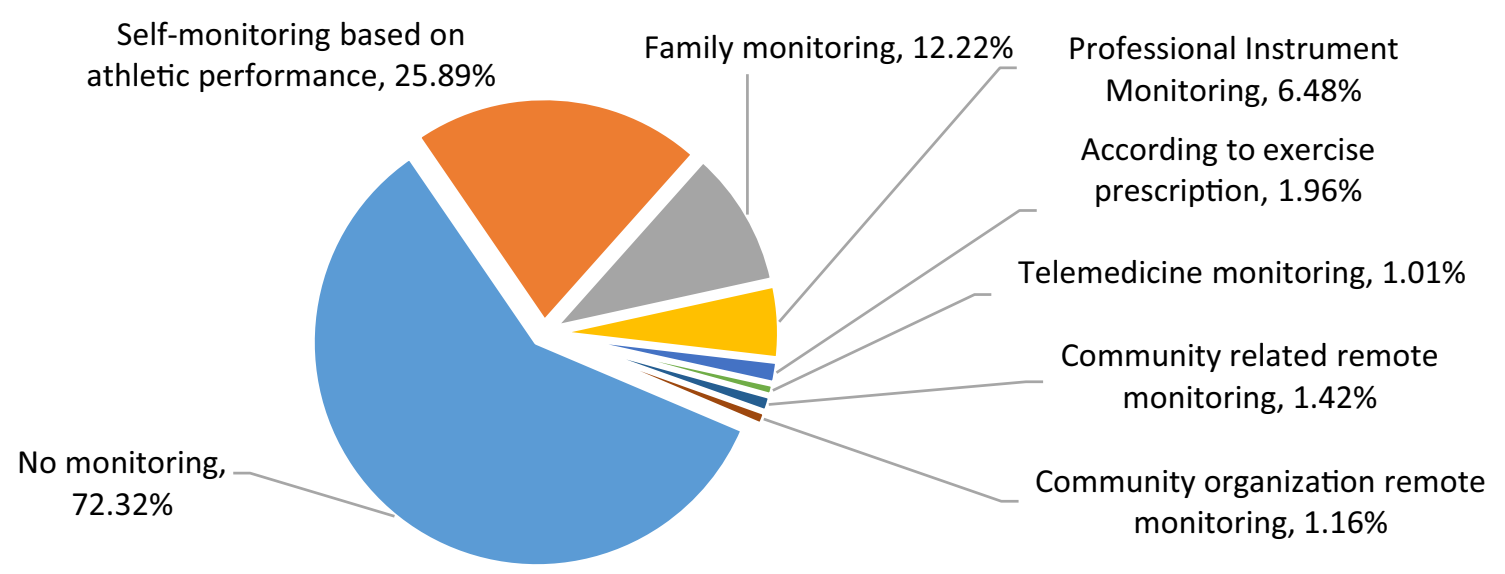

Fig. 7 Pie chart of PA monitoring of Chinese residents during the COVID-19 outbreak

Zhumadian City; Hefei City, Fuyang City, Anhui Province; Nanchang City, Jiangxi Province. Therefore, this paper analyzes the relationship between the insufficiency rate of PA and the epidemic infection rate in the above cities. Pearson correlation coefficient was $-0.467, R^{2}=0.004$, and SIG value was $0.19>0.05$, indicating that there was no significant correlation between the two groups of variables, as shown in Fig. 4. So the infection rate of COVID19 in the city had no significant influence on the PA inadequacy rate.

In addition, during the COVID-19 outbreak period, the PA insufficiency rates of urban residents and rural residents in China were $70.25 \%$ (95\% CI 64.3\%-76.8\%) and 69.86\% (95\%CI 62.9\%-77.2\%), respectively, without significant difference $(p=0.8)$. So there was no significant regional difference in the PA insufficiency rate. Among them, the factors affecting PA of urban and rural residents are basically the same, mainly including limited places and facilities for exercise, lack of exercise partners, atmosphere, mood, etc., as shown in Fig. 5.

\subsection{Overall analysis of PA in different groups}

According to the ANOVA of weekly exercise time of people with different education levels, as shown in Table 6 , the level of education level is negatively correlated with the rate of PA insufficiency $(r=-0.944, p=0.005)$. The weekly PA time of those with bachelor's degree and master's degree and above was the longest during the COVID-19 outbreak period, and the rate of PA insufficiency was $57.93 \%$ (95\% CI $45.64 \%-68.75 \%$ ) and $56.28 \%$ (95\%CI $46.38 \%-78.94 \%)$, respectively. The weekly PA time of those with primary school degree and below (including those without the education) was the shortest. The
Fig. 8 Column chart of PA pattern of Chinese residents during COVID-19 outbreak

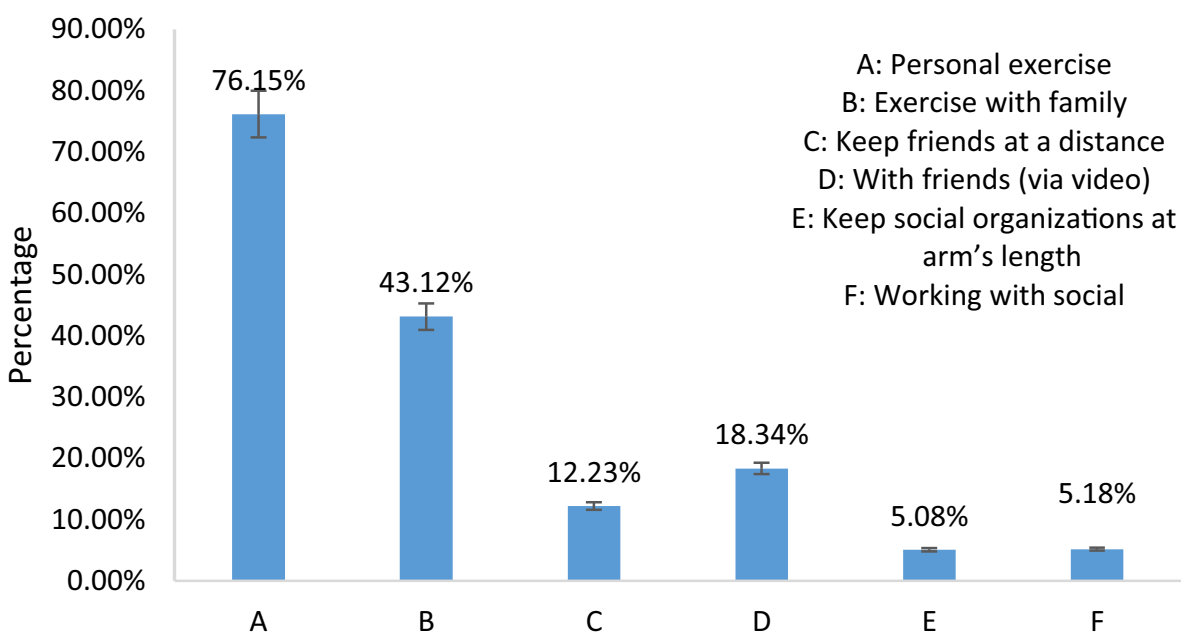


insufficiency rate of PA was as high as $90.65 \%(95 \% \mathrm{CI}$ $86.2 \%-92.4 \%$ ).

In addition, the insufficiency rate of PA presented a situation of "high in the middle and low at both ends", as shown in Fig. 6. The highest insufficiency rate of PA was found in people aged 25-44 $\left(x^{2}=154, p<0.0001\right)$, especially in people aged $30-44\left(x^{2}=126, p<0.0001\right)$. People aged 24 and under $\left(x^{2}=58, p<0.0001\right)$ and over $55\left(x^{2}=264, p<0.0001\right)$ had lower rates of PA insufficiency (Table 7).

\subsection{Analysis of PA scheme}

\subsubsection{Exercise content}

People of different ages have certain differences in the PA content and the source of PA content $\left(x^{2}=88.597\right.$, $p=0.000$ ), as shown in Tables 8 and 9. Children aged 17 and below mainly learn online videos, focusing on family games, rope skipping, shuttlecock, walking or running, home aerobic exercise, and other relatively easy recreational exercises. For 18-24 years old, their exercise content mainly comes from learning network videos, recommendation from friends, etc., and mainly includes aerobics, fighting, fitness equipment, and motion-sensing games, which are obviously in fashion. From the perspective of participating in and urging children to exercise, the exercise content of people aged 25-44 may be highly similar to the main exercise content of children aged 17 and below, with a certain degree of obedience and dependence. From 45 to 54 years old, the exercise content is mainly based on local materials and random cooking combined with online video learning and practicing, focusing on home-based aerobic exercise and freehand exercises, etc., which is generally diversified and somewhat random 55 years old or above, relatively participate in a certain PA social organization or establish their own PA circle of friends, the exercise content is relatively fixed, mainly from the usual exercise habits, specifically focused on the traditional Chinese sports (Taijiquan, Qigong), walking or walking, dancing and other aspects.

\subsubsection{Exercise intensity}

During the COVID-19 outbreak period, there were significant differences in the PA intensity levels of Chinese residents of different ages $(p<0.05)$. In terms of frequency statistics, moderate intensity $(47.69 \%, 95 \% \mathrm{CI}$ $45.25 \%-51.46 \%)$ and low intensity $(40.88 \%$, 95\%CI $39.78 \%-49.24 \%$ ) accounted for the vast majority of the population. Among them, about 20\% (95\%CI 15.26\%$26.15 \%$ ) of people aged 24 and below took strenuous exercise. In addition, there was a significant increase in the proportion of people over 60 years of age who participated in low-intensity exercise $(62 \%, 95 \%$ CI $59.54 \%-64.12 \%)$.

\subsubsection{Time and frequency of exercise}

In general, the duration of a single PA is mainly less than $30 \mathrm{~min}(36.9 \%, 95 \% \mathrm{CI} 32.54 \%-43.26 \%)$ and $30-45 \mathrm{~min}$ $(30.17 \%, 95 \%$ CI $12.89 \%-23.16 \%)$, as shown in Table 10 , showing that the longer the duration of a single PA, the smaller the proportion of the population. Among them, the single exercise time of people aged 24 and below was distributed in different duration periods of 0-90 $\mathrm{min}$ in the adolescent population. The exercise time of people over 60 years old is relatively fixed, generally focusing on $30-60 \min \left(x^{2}=158, p<0.0001\right)$. The frequency of weekly exercise was mainly 3-4 times per week $\left(x^{2}=46\right.$, $p<0.0001$ ), with a high fluctuation, among which the frequency and duration of exercise for people over 60 years old were relatively stable, as shown in Table 11.

\subsubsection{Exercise patterns and monitoring}

In the outbreak period of COVID-19, the PA of Chinese residents was mainly not monitored $(72.32 \%$, 95\% CI $69.45 \%-74.26 \%$ ) or subjective monitoring of self-perception $(25.89 \%$, 95\% CI 22.26\%-28.15\%), as shown in Fig. 7, professional monitoring $(12.22 \%, 95 \%$ CI $10.45 \%-15.81 \%)$ was not well promoted and applied, and community monitoring $(1.42 \%, 95 \%$ CI $1.05 \%-1.89 \%)$, or specialized medical $(1.01 \%, 95 \%$ CI $0.68 \%-1.64 \%)$ telemedicine monitoring was rare. Chinese residents exercise in a single form, mainly by individuals $(76.15 \%$, 95\%CI $71.26 \%-$ $79.46 \%)$ and with their families $(43.62 \%, 95 \%$ CI $40.56 \%-$ $46.28 \%$ ), as shown in Fig. 8, social organizations or associations do not give full play to the network of the public sports service function.

\section{Discussion}

\subsection{Analysis of the general state of PA}

During the outbreak period of COVID-19, the amount of PA of Chinese residents decreased sharply, and the rate of PA insufficiency was about $70 \%$, which was more than twice the global rate. Wuhan took the lead in implementing measures such as home isolation and city closure, while other cities implemented measures such as banning tourism and maintaining social distance, in the early stage of the outbreak (the first week). The rate of insufficient PA among residents in mainland China was $57.5 \%$ [11]. However, with the full-scale outbreak and aggravation of the epidemic, more and more cities practice home isolation and 
close schools in China, which further induces people to panic and other adverse psychological conditions [23], seriously affecting people's normal life style and daily PA [24]. This may be the main reason why most people (including regular exercise) further reduce their daily PA [25]. Meanwhile, the people sedentary, screen time increases sharply, which also leads to the reduction of PA, obesity [26], myopia, and other problems. For example, during the period of epidemic prevention (from January to June in 2020), the myopia rate of primary and middle school students increased by $11.7 \%$ in China, and the intensity of myopic students participating in PA was significantly lower than that of non-myopic students [27] (Ministry of Education 2010). The "SARS" event has set off a national fitness fever [28]. Taking the prevention and control of COVID-19 as a starting point. It is a subject worth thinking about how to strengthen people's fitness consciousness and actions. In general, it can meet the emergency needs of the major public health crisis and strengthen the top-level driving and professional guidance to the national home PA at the national institutional level, which is very important to promote the health of Chinese residents. For example, could develop PA guidelines for different groups in isolation and exercise strategies for full recovery during the post-epidemic period.

\subsection{Analysis of exercise status in different areas}

Regional aspects, there is no significant correlation between the severity of the epidemic situation in provinces and cities and the insufficient rate of PA of residents, and there is no significant difference between urban and rural PA. Fei Qin et al. [11] investigated the PA status in the early stage of the outbreak on the Chinese mainland from January 24 to February 2, 2020 and obtained similar results. The development of rural sports has always been a weak link in the development of sports in China [29]. First of all, the outbreak period of COVID-19 occurred during the traditional Chinese Spring Festival when they went back to visit their families and students' winter vacation. There were a large number of urban populations and young students isolated in rural areas, which might have played a certain role in driving home exercise in rural areas. Secondly, with the implementation of the strategy of rural revitalization and precision poverty alleviation, rural sports facilities have been greatly improved, which has greatly improved the level of health awareness of rural residents and their daily PA habits [30]. The gap between urban and rural residents' health cognition gradually narrowed [31]. Besides, during the COVID-19 pandemic, urban and rural residents mainly did PA at home and both faced common problems such as lack of exercise facilities, which may be the main reason for no significant difference in the rate of
PA insufficiency between urban and rural residents. In addition, climate characteristics, geographical or cultural differences, society-economic levels, and education might affect engagement in PA [11]. Visible, strengthen the construction of indoor sports facilities in the family, to promote PA will have important value.

\subsection{Analysis of exercise status of people with different educational levels}

Educational level is positively related to PA consciousness. Relevant studies show that residents with lower educational levels have less exercise behaviors, and the main reason may be that residents with lower educational levels have less fitness knowledge and less fitness awareness [32]. The survey communique of China's national physical activities shows that people with higher education levels have a higher level of health education, are more willing to participate in PA, and generally have a higher awareness of health [11]. Clearly, the levels of education directly affect the residents' fitness awareness, which may be the important factors for residents to participate in sports activities during the period of new crown pneumonia [33]. Therefore, it is urgent to improve the national fitness level by further improving the overall education level, setting up the national health awareness throughout the life cycle, enhancing the national fitness awareness, changing the health-oriented health thinking pattern, stimulating people's endogenous motivation and independent demand for PA.

\subsection{Analysis of PA at different age}

Different ages have different rates of participating in PA, showing an obvious situation of "high at both ends and low in the middle", the inadequacy rate of people aged 24 and below and over 55 is low, while the inadequacy rate of people aged 25-54 is obviously high. The same result was also reported in the "Survey on the Status of Mass Sports in China" [34]. People under 24 years old have a lower rate of physical inactivity. China implemented "no classes, but no stop studying" strategy during the COVID-19 and the implementation of the online sports practice class. At the same time, social media (WeiBo, WeChat, trill and short video platform) may also play an important role in promoting young people's health behavior [11]. To some extent, the total amount of PA of young children during home epidemic prevention is guaranteed. In addition, parents' perceptual ability plays an important role in promoting PA behavior in adolescents and children [35]. A good family co-parenting environment and ecosystem is an important ecological mechanism for adolescents and children to strengthen PA and promote healthy mental growth 
during the COVID-19 outbreak period [36]. During COVID-19, family members personally participated in children's educational games and online course learning, which effectively stimulated, guided and supervised adolescents' home PA [25]. The people aged between 26 and 55 are the main force to promote the development of social productivity, and the scale, form and behavior of sports activities of these people will have a very significant impact on the whole national fitness [31]. During the outbreak, especially among the 30-44 years old, the group was seriously inadequate in PA. Busy work, less leisure time, heavy burden of life and other problems, resulting in the lack of daily sports fitness awareness and fitness behavior of the crowd [27]. In addition, the multiple tasks such as taking care of and educating children during COVID-19 may be the main reason for the serious lack of PA for this population. It can be seen that although the education level and health awareness of middle-aged people are constantly improving [34], how to transform their internal fitness awareness into practical fitness behavior is an important problem to be solved urgently in realizing national fitness in China.

The people over 55 years old have a relatively low lack of PA, and they mainly prefer low-intensity exercise. The frequency and duration of exercise are relatively stable, they have relatively fixed exercise habits and internal demand drives, and they mainly exercise in a walk with vigorous strides and traditional sports. During the epidemic, meanwhile, they are less affected by the restrictions on exercise places and facilities. It can be seen that the normal exercise habits are the key factors to ensure good PA for the elderly during epidemic prevention. In China, the aging of the population and the aging of the elderly are gradually deepening; the health of the elderly has become the key to the construction of a healthy China. In 2019, the CPC Central Committee and the State Council issued the "Medium- and Long-term Plan for the State to Actively Respond to the Aging of the Population", and the elderly sports have been widely carried out [34], which has played an important role in promoting the elderly to strengthen PA and realize healthy aging. According to the relevant research, the sports consciousness of the elderly is enhanced, the number of participants in sports organizations is increased, and the sports exchange activities of the elderly in the interval are increased [37].

\subsection{Analysis of PA program}

During the COVID-19 pandemic, most people's PA was limited and their exercise mode was forced to change [38], and $48.9 \%$ of them had negative changes in PA [9]. During the period of Chinese residents' home epidemic prevention, the selection of exercise items is diversified, and young people tend to be entertainment and fashion items according to their interests and hobbies; the content of exercise for middle-aged people is arbitrary, and the exercise items for the elderly are the same as daily exercise and relatively fixed. As can be seen, interest, entertainment, exercise habits seem to be the main factors that affect people's choice of exercise content at home isolation [34]. But on the whole, people exercise at home relatively single content. Focusing on aerobic exercise, strength and resistance exercises, static and dynamic stretching and agility, and other comprehensive combinations of exercises, it is possible to achieve greater overall health effects. Among them, teenagers' daily exercise content is mainly learned and practiced through online videos, and the fitness means and skills taught by schools have not been turned into teenagers' daily exercise behaviors. According to the statistics of the National Health and Health Commission in 2017, the health literacy level of young people in China is $15.58 \%$, which is at a low level [39]. The COVID-19 period is a good opportunity to strengthen the health education of adolescents and children [40]. Therefore, from home exercise in the outbreak period to community exercise in the "post-epidemic period", attention should be paid to the integration of children's daily exercise and school physical education, so as to realize the organic docking of family and community exercise and school students' physical literacy cultivation, such as physical awareness, healthy behavior, sports skills, sports morality, etc. Further, build "school—community-family-individual" four-in-one pattern and promotion mechanism of youth physical literacy education. At the exercise load, the main intensity is moderate, and the frequency of a single time is generally lower than $45 \mathrm{~min}$ in a week, which is mainly 3-4 times per week. In general, the short duration of a single session and the low frequency of weekly exercise are the main reasons for insufficient PA.

The form of exercise is the key to creating a happy exercise and the external drive to exercise. During the outbreak period of COVID-19, Chinese residents mainly exercised by themselves or with their families, while social organizations and community public services played little role. Strengthen community, sports association, sports organization, the professional service platform of remote visualization such as public health system was established, through a variety of network visualization in the form of a collective exercise, can provide people with emotional communication and sharing, multivariate evaluation support, such as a good exercise environment, is also an effective way to eliminate the epidemic prevention that occupies the home bad mood [41]. This may be the main task of China's public sports service system in the near future. 
Scientific monitoring is the key to ensuring scientific PA and reducing sports injury. During the outbreak of COVID19 , home isolation destroyed the routine monitoring and guidance system for people's PA [25], and the process of PA in Chinese residents was generally non-monitoring, while in the post-epidemic period, sudden death with masks occurred. It can be seen that the lack of scientific and comprehensive exercise monitoring during the COVID-19 pandemic has seriously affected people's exercise effect or easily caused sports injuries and other accidents. Therefore, building monitoring and support among family members, community and friends support and guidance of network, the public health system and a professional platform of remote health services such as multivariate guiding and risk prevention and control system and dynamic monitoring mechanism, has become a home isolation improves people's PA effect and avoids the key security-related risks. For example, we can use blockchain, Internet and other technologies to establish a public service system for remote guidance and monitoring of PA at home, and promote the remote intelligence and digitization of national fitness services, which may be an important link in the national health promotion system to be improved. Furthermore, although there has been considerable progress in the research and application of exercise prescription in China, it is not systematic and complete [42]. Therefore, the formulation and implementation of standardized, systematic and diversified exercise prescription is the realistic demand to promote the health of the whole people in China, and it is also the key to guide and promote scientific PA during major public health crises such as SARS and COVID-19.

\subsection{Limitations and prospect of research}

Our study had several limitations. Firstly, due to the large population in China, the population of different provinces and cities also varies. The questionnaire survey was randomly distributed through community social platforms in various provinces and cities, WeChat and other Internet platforms to ensure that residents from different regions of the country participated in the survey, and the diversity and representation of the participants were expanded as far as possible. However, due to the network survey, there is a certain age and regional distribution imbalance, in which students (under 25 years old) are relatively more participants, the elderly over 60 years old are relatively less; Hubei, Hunan and other provinces have more participants, while Xinjiang and Heilongjiang have fewer participants. Secondly, although we chose a WHO-approved PA questionnaire (IPAQ-S) with high reliability and validity, the use of participants' self-reported recollection of PA intensity during the pandemic was not as accurate as intensity testing. Thirdly, it is necessary to carry out a comparative analysis of the PA status of the respondents before and after the outbreak of the epidemic, so as to further reveal the specific impact of the epidemic on the PA of the participants and the changing trend of People's Daily physical activities with the development of the epidemic. Finally, it is necessary to follow up with the participants to further reveal the comprehensive effects of PA on epidemic prevention and the promotion of people's physical and mental health during epidemic prevention and to explore the PA programs that may achieve comprehensive health effects during epidemic prevention. Despite the above limitations, this study investigated PA and possible problems in 31 provinces in China during the COVID-19 pandemic phase, which could guide future policies and planning to promote health during major public health events.

\section{Conclusion}

In summary, during the COVID-19 outbreak, nearly $70 \%$ of Chinese residents were not physically active enough, more than double the global rate. Among them, 30-44 years old people have the highest rate of PA insufficiency. There was no significant correlation between PA deficiency rate and epidemic infection rate, but a negative correlation with residents' education level. There was no significant regional difference in the PA insufficiency rate. Nearly $70 \%$ of Chinese residents did not use relevant equipment for PA monitoring during the pandemic. The PA types, intensity, frequency, and duration were different between different ages. Short exercise time per time $(\leq 45 \mathrm{~min})$ and less exercise times per week are the main reasons leading to the rate of the insufficient body weight. The lack of exercise facilities and cultural environment at home and the forced change of exercise methods have seriously affected the PA of Chinese residents. Differences as a function of PA were large relative to other demographic variables such as education, age, and exercise habits. During the period of home isolation, the emphasis should be placed on strengthening the remote network services of social organizations and public service systems, as well as scientific monitoring and guidance of the process of home exercise.

Author contributions Conceptualization, Y.N, Y.M; methodology, Y.N, and Y.; formal analysis, Y. M, and Y.W; investigation, Y.W, W.L, Z.T, C.Z, and J.L; resources, X.L, Y.N; data duration, Z. T, W.L; writing-original draft preparation, Y.N, Y. M and Y.W; writing-review and editing, X.L, T.L, C.L, and C.Z.; supervision, X.L, Y.N; project administration, X.L, Y.N, Y.M. All authors have read and agreed to the published version of the manuscript. 
Funding This research was funded by the National Social Science Foundation of China (grant number 18BTY112), and the young and middle-aged research team of Wuhan Sports University (grant number 21KT13).

\section{Declarations}

Conflict of interest Yingjun Nie and Yuanyan Ma contributed equally to this work. We all declare that we have no conflict of interest in this paper.

\section{References}

1. World Health Organization (2021) WHO coronavirus disease (COVID-19) dashboard with vaccination data. WHO COVID-19 Homepage. https://covid19.who.int/region/wpro/country/cn

2. Weimo Z (2020) Anti-epidemic aerobics. People's Sports Press, 1-2, https://doi.org/10.3390/su12135454

3. Hammami A, Harrabi B, Mohr M, Krustrup P (2020) Physical activity and coronavirus disease 2019 (covid-19): specific recommendations for home-based physical training. Manag Sport Leisure. https://doi.org/10.1080/23750472.2020.1757494

4. Zaka A, Shamloo SE, Fiorente P (2020) COVID19 pandemic as a watershed moment: a call for systematic psychological health care for frontline medical staff. J Health Psychol 7:883-887

5. Zandifar A, Badrfam R (2020) Iranian mental health during the COVID-19 epidemic. Asian J Psychiat 51:101990

6. Jiménez-Pavón D, Carbonell-Baeza A, Lavie CJ (2020) Physical activity as therapy to fight against the mental and physical consequences of COVID-19 isolation: special focus in older people. Prog Cardiovasc Dis 63(3):386-388

7. Violant-Holz V, Gallego-Jiménez MG, González-González CS, Muñoz-Violant S, Rodríguez MJ, Sansano-Nadal O, Guerra-Balic M (2020) Psychological health and physical activity levels during the covid-19 pandemic: a systematic review. Int J Environ Res Public Health 17(24):9419

8. Nienhuis L (2020) The impact of covid-19 on women's physical activity behavior and mental well-being. Int J Environ Res Public Health 17(23):9036

9. Denyer SG (2020) Chinese Coronavirus Infections, Death Toll Soar as Fifth Case Is Confirmed in U.S. Available online: https:// www.washingtonpost.com/world/asia_pacific/coronavirus-chinalatest-updates/2020/2001/2026/4603266c-4603263fa46032684603211ea-afe4603262-4603090eb4603237b4603260b4603 261_story.html Accessed 1 Mar 2020

10. Ammar A, Brach M, Trabelsi K et al (2020) Effects of COVID19 home confinement on eating behaviour and physical activity: results of the ECLB-COVID19 international online survey. Nutrients 12(6): 1583

11. Qin F, Song Y, Nassis GP, Zhao L, Dong Y, Zhao C, Feng Y, Zhao J (2020) Physical activity, screen time, and emotional wellbeing during the 2019 novel coronavirus outbreak in China. Int J Environ Res Public Health 17(14):5170

12. Owen N, Sparling H, Dunstan M (2010) Sedentary behavior: emerging evidence for a new health risk. Mayo Clin Proc 85(12):1138-1141

13. Stanton R, Khalesi S, Williams SL, Alley SJ, Thwaite TL (2020) Depression, anxiety and stress during covid-19: associations with changes in physical activity, sleep, tobacco and alcohosl use in australian adults. Int J Environ Res Public Health 17(11):4065

14. Pinto AJ, Dunstan O et al (2020) Combating physical inactivity during the COVID-19 pandemic. Nat Rev Rheumatol 16(7):1-2
15. Xiang YY, Li W et al (2020) Timely mental health care for the 2019 novel coronavirus outbreak is urgently needed. Lancet Psychiat 7(3):228-229

16. Yang S, Ko C (2020) The mental health burden of the covid-19 pandemic on physical therapists. Int J Environ Res Public Health 17(10):3723

17. Spencer L, Lawrence E (2020) A scoping review of how income affects accessing local green space to engage in outdoor physical activity to improve well-being: implications for post-covid-19. Int J Environ Res Public Health 17(24):9313

18. Lesser N (2020) The impact of covid-19 on physical activity behavior and well-being of canadians. Int J Environ Res Public Health 17(11):3899

19. Fifield A (2020) Travel Ban Goes into Effect in Chinese City of Wuhan as Authorities Try to Stop Coronavirus Spread. Available online: https://www.washingtonpost.com/world/asia_pacific/ninedead-as-chinese-coronavirusspreads-despite-efforts-to-containit/2020/01/22/1eaade72-3c6d-11ea-afe2-090eb37b60b1_story. html. Accessed 1 Mar 2020

20. Lee Yu, Mcdowell L, Lam S (2011) Performance of the international physical activity questionnaire (short form) in subgroups of the hong kong chinese population. Int J Behav Nutr Phys Act $8(1): 81$

21. Sjostrom (2020) Guidelines for Data Processing and Analysis of the International Physical Activity Questionnaire-IPAQ (GDPAIPAQ). Available online: https://www.academia.edu/5346814/ Guidelines_for_Data_Processing_and_Analysis_of_the_Interna tional_Physical_Activity_Questionnaire_IPAQ_Short_and_Long_ Forms_Contents. Accessed 1 Mar 2020

22. WHO (2020) How much of physical activity is recommended? Available online: https://www.who.int/newsroom/fact-sheets/ detail/physical-activity. Accessed 1 Mar 2020

23. Wang P, Wan $T$ (2020) Immediate psychological responses and associated factors during the initial stage of the 2019 coronavirus disease (covid-19) epidemic among the general population in china. Int J Environ Res Public Health 17(5):1729

24. Steffen S, Bastian A, Alexander B et al (2020) Physical activity and screen time of children and adolescents before and during the COVID-19 lockdown in Germany: a natural experiment. Sci Rep 10(1):21780

25. Nie Y, Tan Z, Ma Y, Zhao G (2021) Research progress of physical activity and COVID-19 in Foreign Countries based on knowledge mapping. J Wuhan Sports Univ 55(02):76-84

26. Rundle AG, Park Y, Herbstman JB et al (2020) COVID-19-related school closings and risk of weight gain among Children. Obesity 28(6): 1008-1009

27. Wang D (2020) A press conference of the Ministry of Education on school epidemic prevention and control and education and teaching work in the autumn semester of 2020. http://www.gov. cn/xinwen/2020-08/27/content_5537965.htm.

28. Cao W, Gao C (2003) Situation of people's Physical activity during the period of SARS. Journal Wuhan Sports Univ 05:175-176

29. Lu WY, Lu WZ, Chen ZH (2016) Current situation of village sports public service in integrating urban and rural development and its countermeasures. J Beijing Univ Phys Educ 39(07):1-9

30. Wenyun Lu (2020) Development, experience and prospect of rural public sports services in China. Sports Cult Guide 2020(03):54-61

31. Yang L, Liangjun S (2020) Study on changes in health status of the elderly population based on multidimensional health indicators-Evidence from longitudinal data CLHLS 2002-2014. Northwest Population 41(04):72-89

32. Zhang Y (2007) Discussion on Life Course of Middle Age People in Rural Areas. China Sports Science and Technology 2007(03):47-50 
33. Kari JT, Viinikainen J, Böckerman P, Tammelin TH, Pitkänen N et al (2020) Education leads to a more physically active lifestyle: evidence based on Mendelian randomization. Scand J Med Sci Sports 30(7):1194-1204

34. Yuan-ning P, He J, Tong Y (2000) Sports participation of Chinese people of different ages. Sports Sci 2000(01):23-25

35. Sarto F, Impellizzeri FM, Spörri J (2020) Impact of potential physiological changes due to COVID-19 home confinement on athlete health protection in elite sports: a call for awareness in sports programming. SPORTS MED 50(8):1417-1419

36. Wang H (2012) Family ecosystem study on mental health development of adolescents. Northeastern Univ (philos Soc Sci Ed) 4(06):210-213

37. Ding Z, Zhang X, Yi C (2020) A study on behavioral characteristics, support and influencing factors of physical activity for elderly people in Urban. China Lanzhou J 2020(06):174-187

38. Constandt B, Thibaut E, Bosscher V et al (2020) Exercising in times of lockdown: an analysis of the impact of COVID-19 on levels and patterns of exercise among adults in Belgium. Int $\mathbf{J}$ Environ Res Public Health 17(11):4144

39. Wang J, Hong D (2020) How to release the strength of physical education in epidemic. China Education News. https://www.sohu. com/a/391197441_162758

40. Liu J (2020) Home-based anti-epidemiology is a good opportunity to strengthen health education. China Educ News 2020:4-18

41. Hanke Alexander A, Thorben S, Boeck Hedwig T, Elisabeth S et al (2020) Influence of officially ordered restrictions during the first wave of COVID-19 pandemic on physical activity and quality of life in patients after kidney transplantation in a telemedicine based aftercare program-A KTx $360^{\circ}$ sub study. Int $\mathrm{J}$ Environ Res Public Health 17(23):9144

42. Zhu Li, Wang Z, Zhu W (2020) Construction of sports square library in healthy China vision. Sports Sci 40(01):4-15

Publisher's Note Springer Nature remains neutral with regard to jurisdictional claims in published maps and institutional affiliations. 